\title{
Observation of metastable states in spinor Bose-Einstein condensates
}

\author{
H.-J. Miesner, D.M. Stamper-Kurn, J. Stenger, S. Inouye, A.P. Chikkatur, and W. Ketterle \\ Department of Physics and Research Laboratory of Electronics, \\ Massachusetts Institute of Technology, Cambridge, MA 02139
}

(November, 1998)

\begin{abstract}
Bose-Einstein condensates have been prepared in long-lived metastable excited states. Two complementary types of metastable states were observed. The first is due to the immiscibility of multiple components in the condensate, and the second to local suppression of spin-relaxation collisions. Relaxation via re-condensation of non-condensed atoms, spin relaxation, and quantum tunneling was observed. These experiments were done with $F=1$ spinor Bose-Einstein condensates of sodium confined in an optical dipole trap.
\end{abstract}

Metastable states of matter, excited states which relax only slowly to the ground state, are commonly encountered. This slow relaxation often arises from the presence of free-energy barriers that prevent a system from directly evolving toward its ground state; if the thermal energy to overcome this barrier is not available, the metastable state may be long-lived.

Many properties of Bose-Einstein condensates in dilute atomic gases [1] arise from metastability; indeed, such condensates are themselves metastable, since the true equilibrium state is a solid at these low temperatures. Bose-Einstein condensates in gases with attractive interactions (scattering length $a<0$ ) [3 are metastable against collapse due to a kinetic energy barrier [5]. The persistence of rotations in condensates with repulsive interactions $(a>0)$ hinges on whether vortices are metastable in singly- [6] or multiply-connected [7], 8] geometries. Similarly, dark solitons in restricted geometries are predicted to be long-lived [9] akin to the recently observed metastable states in superfluid ${ }^{3} \mathrm{He}-$ $\mathrm{B}$ [10]. Finally, Pu and Bigelow discussed spatial distributions of two-species condensates which are metastable due to mean-field repulsion between the two species [11.

In this Letter, we report on the observation of two complementary types of metastability in $F=1$ spinor Bose-Einstein condensates of sodium. In one, a twocomponent condensate in the $\left|F=1, m_{F}=1,0\right\rangle$ hyperfine states was stable in spin composition, but spontaneously formed a metastable spatial arrangement of spin domains. In the other, a single component $\left|m_{F}=0\right\rangle$ condensate was metastable in spin composition with respect to the development of $\left|m_{F}= \pm 1\right\rangle$ ground-state spin domains. In both cases, the energy barriers which caused the metastability (as low as $0.1 \mathrm{nK}$ ) were much smaller than the temperature of the gas (about $100 \mathrm{nK}$ ) which would suggest a rapid thermal relaxation. However, this thermal relaxation was slowed considerably due to the high condensate fraction and the extreme diluteness of the non-condensed cloud.

Spinor Bose-Einstein condensates were created as in previous work $\left[12 \mid\right.$. Condensates in the $\mid F=1, m_{F}=$ $-1)$ state were created in a magnetic trap 13 and then transfered to an optical dipole trap formed by a single infrared laser beam [14]. The beam was weakly focused, producing cigar-shaped traps with depths of $1-2 \mu \mathrm{K}$, radial trap frequencies of about $500 \mathrm{~Hz}$, and aspect ratios of about 70. After transfer, Landau-Zener rf-sweeps placed the optically trapped atoms into the desired initial spin states. Condensates were then held for as long as $20 \mathrm{~s}$ during which time the condensate numbers decayed from about $10^{6}$ to $10^{5}$. Condensate densities ranged from about 5 to $1 \times 10^{14} \mathrm{~cm}^{-3}$.

The spin-state distribution along the long axis of the optical trap was probed by a Stern-Gerlach spin separation combined with time-of-flight imaging [12]. After a sudden switch-off of the optical trap, the condensate expanded primarily radially. After $5 \mathrm{~ms}$, a magnetic field gradient of several $\mathrm{G} / \mathrm{cm}$ separated the different spinstate populations without distorting them. Axial striations observed in time-of-flight images therefore reflected the spatial structure of the trapped condensate. After a total time-of-flight of $15-30 \mathrm{~ms}$, the atoms were optically pumped to the $\left|F=2, m_{F}=2\right\rangle$ hyperfine state, and imaged with resonant light.

For our first set of experiments, a condensate in a superposition of the $\left|F=1, m_{F}=1,0\right\rangle$ hyperfine states was prepared by first transferring all the atoms to the $\left|m_{F}=1\right\rangle$ state with an adiabatic rf-sweep at low field $(1 \mathrm{G})$, and then transferring half of the population to the $\left|m_{F}=0\right\rangle$ state with a non-adiabatic sweep at high field $(15 \mathrm{G})$. The sample then evolved freely in a $15 \mathrm{G}$ bias field which prevented the formation of $\left|m_{F}=-1\right\rangle$ spin domains due to the large quadratic Zeeman energy barrier [12].

Following their preparation, the two spin components began to separate radially (Fig. 1, sideways in images). Within $50 \mathrm{~ms}$, axial density striations were observed, indicating the formation of $\left|m_{F}=1\right\rangle$ and $\left|m_{F}=0\right\rangle$ spin domains. The striations were initially angled due to radial excitations which tilted the domain walls at various 
angles to the long axis of the cloud. After about $100 \mathrm{~ms}$, the striations became horizontal, indicating the dissipation of radial motion and the orientation of the domain walls perpendicular to the axis of the cloud. The spin domains reached typical sizes of $40 \pm 15 \mu \mathrm{m}$, slightly larger than their size after $50 \mathrm{~ms}$. Thereafter, the clouds were essentially unchanged - only the total number of atoms diminished due to three-body losses. Matching the images of the $\left|m_{F}=1\right\rangle$ and $\left|m_{F}=0\right\rangle$ spin distributions showed that these long-lived states consist of persistent, alternating $\left|m_{F}=1\right\rangle$ and $\left|m_{F}=0\right\rangle$ spin domains.

Phase-separation in two-component condensate mixtures is predicted by mean-field theory 15 19. The mean-field interaction energy of such condensates is given by $2 \pi \hbar^{2} / m \times\left(n_{a}^{2} a_{a}+n_{b}^{2} a_{b}+2 n_{a} n_{b} a_{a b}\right)$ where $m$ is the common atomic mass, $n_{a}$ and $n_{b}$ are the densities of each of the components, $a_{a}$ and $a_{b}$ are the same-species scattering lengths, and $a_{a b}$ is the scattering length for interspecies collisions. The mean-field energy is minimized by phase-separation when the scattering lengths obey the relation $a_{a b}>\sqrt{a_{a} a_{b}}$. At low magnetic fields, the scattering lengths in the $F=1$ spinor system are determined by $a_{F_{t o t}=0}$ and $a_{F_{t o t}=2}$ which describe collision between atoms with total angular momentum $F_{t o t}=0,2$ 20]. Defining $\bar{a}=\left(2 a_{F_{t o t}=2}+a_{F_{t o t}=0}\right) / 3$ and $\Delta a=\left(a_{F_{t o t}=2}-a_{F_{t o t}=0}\right) / 3$, the scattering lengths for the $\left|m_{F}=1,0\right\rangle$ two-component system are given by $a_{0}=\bar{a}$, and $a_{1}=a_{01}=\bar{a}+\Delta a$. Since $\Delta a$ was measured to be $3.5 \pm 1.5 \mathrm{bohr}>0$ [12], $a_{01}>\sqrt{a_{0} a_{1}}$ and the components phase-separate. Interestingly, this phase-separation should not occur in the non-condensed cloud because the same-species mean-field interaction energies are doubled due to exchange terms.

The tendency of a two-component mixture to phaseseparate implies the presence of imaginary frequencies for out-of-phase collective excitations of the overlapping components (spin waves) 19,21. This means that the condensates are unstable, and slight perturbations grow exponentially. In our experiment, density and magnetic field inhomogeneities may yield slight perturbations in the initial state, corresponding to an initial distribution of spin wave excitations. The spontaneous formation of spin domains thus constitute an observation of spin waves with imaginary frequencies at wavelengths of $10-50 \mu \mathrm{m}$, corresponding to the size of the spin domains which are intially formed.

The many-domain spin distribution is a macroscopically occupied excited state; the ground state of the twocomponent system would contain only one domain each of $\left|m_{F}=0\right\rangle$ and $\left|m_{F}=1\right\rangle$ atoms, minimizing the surface energy of the domain walls [24]. For the many-domain state to decay directly to the ground state, the two condensate components would have to either overlap, or else pass by each other without overlapping. Such motion is energetically forbidden: the former due to the meanfield energy (typically $50 \mathrm{~Hz}$ or $2.5 \mathrm{nK}$ [12]), and the latter due to the large kinetic energy required to vary the condensate wavefunction radially (the condensate is about $5 \mu \mathrm{m}$ wide, yielding a kinetic energy barrier of $50-100 \mathrm{~Hz}$ ). For this reason, the excited states we observe are metastable. In comparison, experiments on two-component mixtures of ${ }^{87} \mathrm{Rb}$ showed no metastability, due in part to the nearly spherical traps (aspect ratio of $1 / \sqrt{8}$ ) which were used [22].

The typical size of the metastable spin domains can be understood by considering the width of the domain walls $(\simeq 3 \mu \mathrm{m}[23])$. Spin domains smaller than this width do not prevent the tunneling of unlike-spin atoms through the domain, and thus small spin domains are free to migrate through the cloud and coalesce with other like-spin domains. Spin domains which are several times larger than the boundary width prevent the tunneling of atoms across the domain, and are thus stable. Metastable domains shorter than $\approx 25 \mu \mathrm{m}$ were not observed, although the above description suggests that domains as short as $\approx 10 \mu \mathrm{m}$ could be stable. This may be due to either an axial smearing of small domains during their free expansion prior to imaging, or perhaps the destabilization of small domains by the initial radial excitations in the trapped cloud.

The spontaneously formed metastable states were extremely long lived. In the absence of an externally applied magnetic field gradient, the states persisted for at least 20 seconds, by which time the condensate number had dropped so low that we were unable to properly assess the time-of-flight images. The lifetime of the metastable state was shortened by applying a magnetic field gradient along the axis of the trap. At field gradients of $0.1 \mathrm{G} / \mathrm{cm}$ or greater, the metastable states decayed to the ground state within experimentally accessible times of 10 seconds or less.

To study this decay with a well-defined initial state, we first created a metastable two-domain system. A strong magnetic field gradient was applied, causing the many spin domains to collapse into the ground state of two domains with the $\left|m_{F}=1\right\rangle$ domain at the high-field end of the cloud. A weaker field gradient $(0.1 \mathrm{G} / \mathrm{cm})$ was then applied in the opposite direction, which energetically favored the rearragement of the spin domains at opposite ends of the cloud. In spite of this gradient, the two-domain system was metastable (Fig. 2). It decayed slowly to equilibrium via two processes: a slow process which dominated for the first $12 \mathrm{~s}$, and a fast process (between $12 \mathrm{~s}$ and $13 \mathrm{~s}$ ) which suddenly depleted the metastable domains.

The slow process caused a gradual increase in the number of atoms in the two ground state spin domains, which saturated at about $5 \times 10^{4}$ atoms in each. The rate of accumulation did not depend strongly on condensate density, which was varied by decreasing the total number of atoms before creating the metastable state. Thus, the slow process is probably due to re-condensation of the di- 
lute thermal cloud into ground state spin domains, driven by a difference $\Delta \mu$ between the chemical potential of the thermal cloud and the energy of the ground state.

Studies of the formation of one-component Bose condensates in harmonic traps [25,26] considered the case $T \gg \mu$ where $T$ is the temperature of the cloud, and $\mu$ is the chemical potential of the condensate $(\mu>0$ due to interactions). For optically trapped Bose condensates, the temperature is about $1 / 10$ of the optical trap depth due to evaporation, i.e. $T \approx 100 \mathrm{nK}$ 14]. For typical densities of $3 \times 10^{14} \mathrm{~cm}^{-3}, \mu=220 \mathrm{nK}>T$. Thus, previous studies of condensate formation may not apply to these metastable states.

The sudden decay to the ground state after the slow relaxation indicated the onset of a faster relaxation mechanism, which occurred once the decaying condensate had reached a critical density. The dependence of this critical density on the applied field gradient suggests the onset of macroscopic quantum tunneling across the metastable spin domains. Such behaviour will be reported in greater detail elsewhere.

The metastable states discussed so far involved multi-component mixtures stable in composition, but metastable in their spatial distribution. Conversely, we identified another form of metastability in which a single component condensate was stable in its spatial distribution, but metastable in its hyperfine composition. This was observed in spinor condensates with an overall spin projection $\left\langle F_{z}\right\rangle=0$ along the magnetic field axis 12]. Condensates were prepared using LandauZener rf-sweeps at high fields (30 G) in one of two ways: either the entire trapped condensate was placed in the $\left|m_{F}=0\right\rangle$ state by a single rf-sweep, or the condensate was placed in a 50-50 mixture of the $\left|m_{F}=+1\right\rangle$ and $\left|m_{F}=-1\right\rangle$ states using two rf-sweeps. The condensates were then allowed to reach the ground state at a magnetic bias field $B_{0}$ and axial field gradient $B^{\prime}$.

The evolution to equilibrium from the two starting conditions was quite different (Fig. 3). When starting from the $\left|m_{F}=0\right\rangle$ state, the condensate remained in that state for about three seconds before evolving over the next few seconds to the ground state. On the other hand, starting from an equal mixture of $\left|m_{F}=+1\right\rangle$ and $\left|m_{F}=-1\right\rangle$ atoms, the fraction of atoms in the $\left|m_{F}=0\right\rangle$ state grew without delay, arriving at equilibrium within just $200 \mathrm{~ms}$.

This difference can be understood by considering a spin-relaxation collision, in which two $\left|m_{F}=0\right\rangle$ atoms collide to produce a $\left|m_{F}=1\right\rangle$ and a $\left|m_{F}=-1\right\rangle$ atom. In the presence of a magnetic field $B_{0}$, quadratic Zeeman shifts cause the energy of the two $\left|m_{F}=0\right\rangle$ atoms to be lower than the $\left|m_{F}=1\right\rangle$ and $\left|m_{F}=-1\right\rangle$ atoms by $2 \times 390 \mathrm{~Hz} \times\left(B_{0} / \mathrm{G}\right)^{2}$. Due to this activation energy, condensate atoms in the $\left|m_{F}=0\right\rangle$ state cannot undergo spin-relaxation collisions. Thus, even though the creation of $\left|m_{F}=1\right\rangle$ and $\left|m_{F}=-1\right\rangle$ spin domains at the ends of the condensate is energetically favored globally in the presence of a magnetic field gradient, the $\left|m_{F}=0\right\rangle$ condensate cannot overcome the local energy barrier for spinrelaxation. On the other hand, condensate atoms in the $\left|m_{F}=1\right\rangle$ and $\left|m_{F}=-1\right\rangle$ states can directly lower their energy through such collisions, and equilibrate quickly.

The decay of the metastable $\left|m_{F}=0\right\rangle$ state was studied by varying the bias field $B_{0}$, the field gradient $B^{\prime}$, and the trap depth $U$. Evolution curves under various conditions (Fig. 4) were characterized by a delay time $T_{d}$ before the fraction of condensate atoms in the $\left|m_{F}=0\right\rangle$ state decayed exponentially to equilibrium with a relaxation time constant $\tau$. Increasing the bias field $B_{0}$ did not affect $\tau$ significantly, but increased the delay time from $T_{d} \simeq 1.9 \mathrm{~s}$ at small bias fields $(55 \mathrm{mG})$ to $T_{d} \simeq 3.6 \mathrm{~s}$ for larger fields $(250 \mathrm{mG})$. Lowering the trap depth from 2 $\mu \mathrm{K}$ to $1.2 \mu \mathrm{K}$ increased both $T_{d}$ from about 0.6 to $4 \mathrm{~s}$, and $\tau$ from about 3.8 to $6.6 \mathrm{~s}$. Increasing the magnetic field gradient only induced a weak lowering of $\tau$, and $T_{d}$ was not affected significantly.

The delay time $T_{d}$ should reflect the time-scale for the gradual accumulation of $\left|m_{F}= \pm 1\right\rangle$ atoms in the thermal cloud before they reach a critical density and condense into the ground-state spin domains. Increasing the bias field increases the quadratic Zeeman energy barrier for spin-relaxation, lengthening $T_{d}$. However, the clear variation of $T_{d}$ as the energy barrier is changed from $0.1 \mathrm{nK}$ at $55 \mathrm{mG}$ to $2.5 \mathrm{nK}$ at $250 \mathrm{mG}$, energies much smaller than the temperature of $T \approx 100 \mathrm{nK}$, cannot be explained on the basis of thermal spin-relaxation collisions. Further study of these relaxation timescales is warranted.

In conclusion, we have identified a novel form of metastability in Bose-Einstein condensates which occurs at temperatures much higher than the energy barrier for evolution to the ground state. This metastability arises due to the high condensate fraction which dramatically slows down thermal relaxation. This offers the opportunity to study the kinetics of condensation and of the dissolution of metastable condensates in "slow motion," on times scales much longer than collision times and of periods of collective excitations. Furthermore, we have observed spontaneous phase-separation in a two-component mixture in accordance with the predictions of mean-field theory.

This work was supported by the Office of Naval Research, NSF, Joint Services Electronics Program (ARO), NASA, and the David and Lucile Packard Foundation. A. P. C. acknowledges additional support from the NSF, D. M. S. -K. from JSEP, and J. S. from the Alexander von Humboldt-Foundation.

[1] M.H. Anderson et al., Science 269, 198 (1995). 
[2] K.B. Davis et al., Phys. Rev. Lett. 75, 3969 (1995).

[3] C.C. Bradley, C.A. Sackett, and R.G. Hulet, Phys. Rev. Lett. 78, 985 (1997), see also: C.C.Bradley et al., Phys. Rev. Lett. 75, 1687 (1995).

[4] D.S. Fried et al., Phys. Rev. Lett. 81, 3811 (1998).

[5] Yu. Kagan, G.V. Shlyapnikov, and J.T.M. Walraven, Phys. Rev. Lett. 76, 2670 (1996).

[6] D.S. Rokhsar, Phys. Rev. Lett. 79, 2164 (1997).

[7] D.S. Rokhsar, preprint cond-mat/9709212.

[8] E.J. Mueller, P.M. Goldbart, and Y. Lyanda-Geller, Phys. Rev. A 57, R1505 (1998).

[9] S.A. Morgan, R.J. Ballagh, and K. Burnett, Phys. Rev. A 55, 4338 (1997); W.P. Reinhardt and C.W. Clark, J. Phys. B 30, L785 (1997); T.F. Scott, R.J. Ballagh, and K. Burnett, J. Phys. B 31, L329 (1998); A.D. Jackson, G.M. Kavoulakis, and C.J. Pethick, Phys. Rev. A 58, 2417 (1998).

[10] S. Backhaus et al., Nature 392, 687 (1998).

[11] H. Pu and N.P. Bigelow, Phys. Rev. Lett. 80, 1134 (1998).

[12] J. Stenger et al., Nature (in press).

[13] M.-O. Mewes et al., Phys. Rev. Lett. 77, 416 (1996).

[14] D.M. Stamper-Kurn et al., Phys. Rev. Lett. 80, 2027 (1998).

[15] W.B. Colson and A.L. Fetter, Jour. Low. Temp. Phys. 33, 231 (1978).

[16] T.-L. Ho and V.B. Shenoy, Phys. Rev. Lett. 77, 3276 (1996).

[17] B.D. Esry, C.H. Greene, J.P. Burke, Jr. and J.L. Bohn, Phys. Rev. Lett. 78, 3594 (1997).

[18] S.T. Chui, B. Tanatar, and P. Ao, preprint.

[19] E.V. Goldstein and P. Meystre, Phys. Rev. A 97, 2935 (1997).

[20] T.-L. Ho, Phys. Rev. Lett. 81, 742 (1998).

[21] R. Graham and D. Walls, Phys. Rev. A 98, 484 (1998).

[22] D.S. Hall et al., Phys. Rev. Lett. 81, 1539 (1998).

[23] Approximating the two condensate wavefunctions in the boundary region $(0 \leq z \leq L)$ to be $\psi_{1}=\sqrt{n_{1}} \cos \pi z / 2 L$, $\psi_{0}=\sqrt{n_{0}} \sin \pi z / 2 L$, and assuming equal pressure $\left(n_{1}^{2} a_{1}=n_{0}^{2} a_{0}\right)$ across the boundary of length $L$, the kinetic and mean-field (see text) energies are minimized at $L^{2}=\pi \xi_{1}^{2} \frac{a_{1}+\sqrt{a_{1} a_{0}}}{a_{01}-\sqrt{a_{1} a_{0}}}$. Here $\xi_{1}=\left(8 \pi n_{1} a_{1}\right)^{-1 / 2}$. For $n_{1} \simeq 3 \times 10^{14} \mathrm{~cm}^{-3}$, and $a_{1}=52 \mathrm{bohr}, L \simeq 3 \mu \mathrm{m}$.

[24] P. Ao and S.T. Chui, preprint cond-mat/9809195.

[25] H.-J. Miesner et al., Science 279, 1005 (1998).

[26] C.W. Gardiner, P. Zoller, R.J. Ballagh, and M.J. Davis, Phys. Rev. Lett. 79, 1793 (1997).

FIG. 1. Spontaneous formation of metastable states in a 15 $\mathrm{G}$ bias field. Condensates were probed at various times after preparing an overlapping $\left|m_{F}=1,0\right\rangle$ mixed condensate. The $\left|m_{F}=1\right\rangle$ (top) and $\left|m_{F}=0\right\rangle$ (bottom) states were separated during their free expansion. Striations reflect the presence of alternating $\approx 40 \mu \mathrm{m}$ spin domains which form $50 \mathrm{~ms}$ after state preparation. High resolution $(\approx 10 \mu \mathrm{m})$ was achieved by only optically pumping a $200 \mu \mathrm{m}$ slice of the clouds. The height of each image is $1.3 \mathrm{~mm}$.

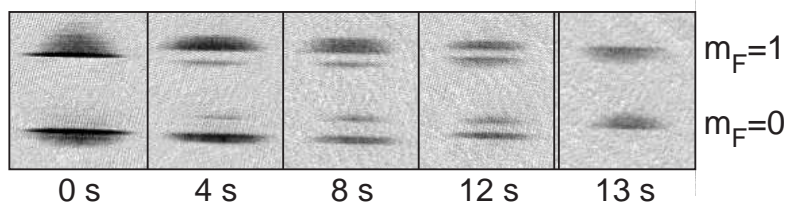

FIG. 2. Relaxation of metastable states in a $15 \mathrm{G}$ bias field and a $0.1 \mathrm{G} / \mathrm{cm}$ gradient. Images show metastable (outer) and ground state (inner) spin domains of $\left|m_{F}=1\right\rangle$ and $\left|m_{F}=0\right\rangle$ atoms, probed at various times after state preparation. The metastable domains decayed slowly for $12 \mathrm{~s}$ before tunneling quickly to the ground state. The height of each image is 1.3 $\mathrm{mm}$.

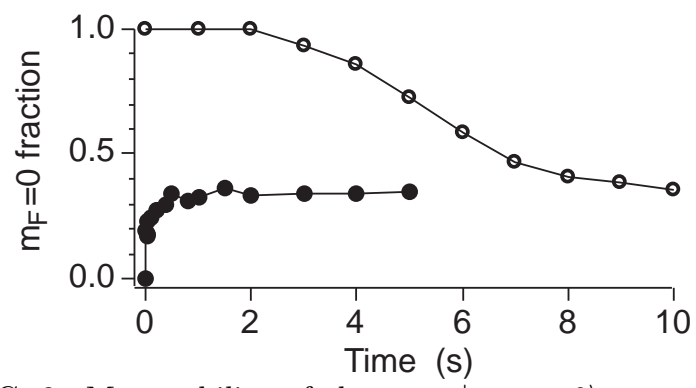

FIG. 3. Metastability of the pure $\left|m_{F}=0\right\rangle$ state in the presence of a magnetic bias field $(250 \mathrm{mG})$, and gradient $(44 \mathrm{mG} / \mathrm{cm})$. The evolution toward equilibrium of a pure $\left|m_{F}=0\right\rangle$ condensate (open symbols), and a mixture of $\left|m_{F}=1\right\rangle$ and $\left|m_{F}=-1\right\rangle$ (closed symbols) is shown by plotting the observed fraction of atoms in the $\left|m_{F}=0\right\rangle$ state vs. dwell time in the optical trap.

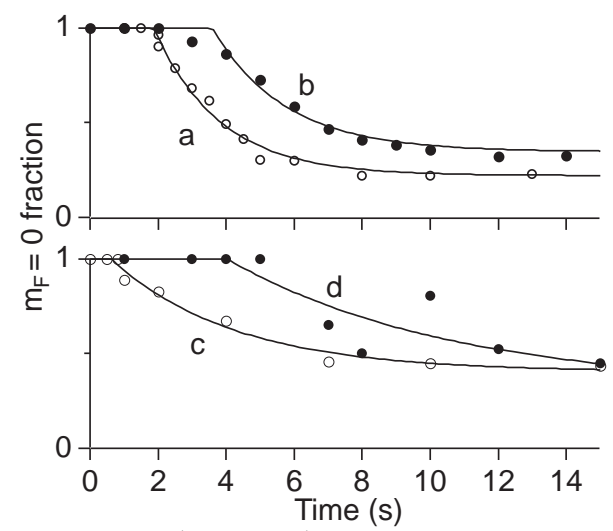

FIG. 4. Decay of $\left|m_{F}=0\right\rangle$ metastable states. The lines are exponential decay fits to the $\left|m_{F}=0\right\rangle$ fraction after a delay time $T_{d}$. Increasing the bias field (top) increased $T_{d}$. Lowering the temperature of the gas (bottom) increased both $T_{d}$ and $\tau$. The magnetic bias field $B_{0}$, the gradient $B^{\prime}$, and the trap depth $U$ for each curve are: a) $55 \mathrm{mG}, 44 \mathrm{mG} / \mathrm{cm}$, $2 \mu \mathrm{K}$; b) $250 \mathrm{mG}, 44 \mathrm{mG} / \mathrm{cm}, 2 \mu \mathrm{K}$; c) $20 \mathrm{mG}, 11 \mathrm{mG} / \mathrm{cm}, 2$ $\mu \mathrm{K}$; and d) $20 \mathrm{mG}, 11 \mathrm{mG} / \mathrm{cm}, 1.2 \mu \mathrm{K}$. 
This figure "msfigure1.jpg" is available in "jpg" format from: http://arxiv.org/ps/cond-mat/9811161v1 